\title{
TL Characteristics and Dosimetric Aspects of Mg-Doped ZnO
}

\author{
Abeer Z. Abraheem, F. Khamis, and Y.A. Abdulla
}

\section{ABSTRACT}

Dosimetry characterization and the evaluation of kinetics parameters of trapping states of Mg-doped $\mathrm{ZnO}$ phosphors synthesized by Sol-Gel technique. The thermoluminescence response of Mg-doped $\mathrm{ZnO}$ samples showed a linear response when exposed to $X$-ray radiation and the optimum annealing condition was $400^{\circ} \mathrm{C} / 4 \mathrm{~h}$ for the three concentrations. A broad-shaped TL glow curve with an upper bound of $270{ }^{\circ} \mathrm{C}$, which shifts to lower temperatures with increasing dose, indicating that general order (GO) kinetics thermoluminescence processes are involved. We conclude that the $\mathrm{ZnO}$ doped $\mathrm{Mg}$ phosphors under study are promises to develop dosimeters for high radiation dose measurements. Kinetic parameters, such as activation energy (E), frequency factor (s), and order of kinematic order (b), were estimated by the Glow Curve Deconvolution (GCD) method. ZnO:Mg phosphor has a great potential as a dosimeter for monitoring in the fields of ionizing radiation.

Keywords: Mg doped ZnO, TL, Sol-Gel Method, GCD.
Published Online: January 29, 2021

ISSN: $2684-4451$

DOI :10.24018/ejphysics.2021.3.1.37

Abeer Z. Abraheem

Department of Physics, Sebha

University, Libya.

(e-mail: Salma2011@gmail.com)

F. Khamis*

Department of Physics, University of

Tripoli, Tripoli 13220, Libya.

(e-mail: f.khamis@uot.edu.ly)

Y.A. Abdulla

Department of Physics, Sebha

University, Libya.

(e-mail: you.omer@sebhau.edu.ly)

*Corresponding Author

\section{INTRODUCTION}

Thermoluminescence (TL) is the emission of light by heating a phosphorus material after absorbing energy from ionizing radiation [1]. The energy emitted, in the form of luminescence, and the sensitivity of photon detection make the TL phenomenon an attractive way to measure small amounts of stored energy. The development of luminescent materials has been successfully applied in various fields and enhanced human life [2]. Applications of the TL phenomenon have been found not only in dosimetry, research, earth sciences, and the age determination of archaeological or geological samples [3]-[7].

$\mathrm{ZnO}$ is a metal oxide nanostructure and has attracted a great deal of attention due to its use in many applications. Recently $\mathrm{ZnO}$ doped magnesium materials have attracted great interest due to their unique properties in UVillumination based on radiative recombination of electron hole pairs [8], [9]. Stoichiometric and homogeneity of composition are key to the potential applications of such mixed metal oxides [10]. $\mathrm{ZnO}$ crystals consist of a hexagonal lattice of decomposition $\left(\mathrm{a}=3.296^{\circ} \mathrm{A}, \mathrm{c}=5.206\right)$, the ionic radius of $\mathrm{Mg}^{+2}\left(0.57^{\circ} \mathrm{A}\right)$ and $\mathrm{Zn}^{+2}\left(0.6^{\circ} \mathrm{A}\right)$ is nearly identical, allowing the substitution of $\mathrm{Mg}$ ion in the resulting sites of zinc solution solid in a wide range of concentrations [11]-[15] so that $\mathrm{ZnO}$ is readily doped with $\mathrm{Mg}$. Under doping, its electrical properties may vary from insulator to semiconductor, depending on the doping used. Moreover, it has been reported that $\mathrm{ZnO}$ exhibits radiative hardness when exposed to ionizing radiation and, in this sense, the material appears to be a good candidate for high dose measurement [16]-[21].

Several models have been applied to describe the TL process that arose in materials. The easiest method, called first-order kinetics, assumes that the thermal release of an electron from the trap centers is followed by recombination with a hole in the recombination centers. In this model, it is assumed that the electron retrapped potential is impossible [22]. While the second-order model considers that there are two possibilities given to the electrons upon the thermal release: recombine with holes or re-trap again [23]. An experimental model has been proposed to describe the situation when first or second order TL peak is satisfied, called general order kinetics [24]. The specific details of the trap and recombination centers provide valuable information about the material's properties, defects, and radiation absorption history of. This information is found in the trap parameters of the centers. Parameters include the activation energy (depth of trap center) $\mathrm{E}(\mathrm{eV})$, the kinetic order of TL processes $b$ (first, second and general), the initial concentrations of trapped charges $\mathrm{n}_{0}\left(\mathrm{~cm}^{-3}\right)$, the frequency factor $\mathrm{s}\left(\mathrm{s}^{-1}\right)$, (escape probability) in the case of exponential factor of first order or pre-exponential factor $\mathrm{s}^{\prime \prime}\left(\mathrm{s}^{-1}\right)$ in the case of second and general order kinetics. Typically, the TL glow curves are analyzed assuming different b-values, i.e., suppose a first, second, or general order model.

In this paper we reported the TL and dosimetric properties of $\mathrm{Mg}$-doped $\mathrm{ZnO}$ synthesized by Sol-Gel technique when exposed to an X-ray Source. 


\section{EXPERIMENTAL}

\section{A. Sample Preparation}

Samples are prepared from $\mathrm{ZnO}$ doped $\mathrm{Mg}$ nanoparticles by Sol-Gel method. Starting materials are zinc nitrate, magnesium nitrate, acetic acid and EDTA. The experiment is performed by dissolving together the equivalent amount of the above reagents with a minimum amount of distilled water. $\mathrm{ZnO}$ nanoparticles prepared in three concentrations $(1 \%, 2 \%, 3 \mathrm{wt} \%)$ the resulting powder is subjected to calcinations at $600{ }^{\circ} \mathrm{C} / 2 \mathrm{~h}$, then $0.1 \mathrm{~g}$ of powder is placed in a cylindrical mold with a diameter $0.85 \mathrm{~cm}$ to press about 1 ton using a hydraulic press to make pellet shaped sample. Dosimetry characteristics of the prepared samples were determined using the Harshaw TL Reader "Model 3500".

\section{RESULTS AND DISCUSSION}

\section{A. Annealing and Reusability}

One of the most important parameters to be investigated for any TLD material, is the annealing procedure. The annealing procedure is required in order to get the highest sensitivity and at the same time to eliminate the effects of the previous irradiation. The characterization annealing is usually done by systematically studying the change of TL sensitivity as a function of temperature $\mathrm{T}$ and time $\mathrm{t}$, at each given temperature. The $(T, t)$ values determined to provide the optimal annealing procedure shall fulfill the following basic requirements: a) the ability to erase all previously accumulated radiation history, b) the ability to produce the highest sensitivity, and c) the ability to maintain stable sensitivity after several cycles of annealing by radiation. The experiment was conducted over a temperature range of $100{ }^{\circ} \mathrm{C}$ to $500{ }^{\circ} \mathrm{C}$ in steps of $100{ }^{\circ} \mathrm{C}$, and annealing time for $1 \mathrm{~h}, 2 \mathrm{~h}, 3 \mathrm{~h}, 4 \mathrm{~h}$, and $5 \mathrm{~h}$ for each set temperature. The operation was performed for the three concentrations. Fig 1 shows the TL response obtained from irradiated samples of $\mathrm{ZnO}$ doped with $1 \%, 2 \%$, and $3 \%$ of $\mathrm{Mg}$. The best annealing procedure was obtained at $400{ }^{\circ} \mathrm{C} / 4 \mathrm{~h}$.

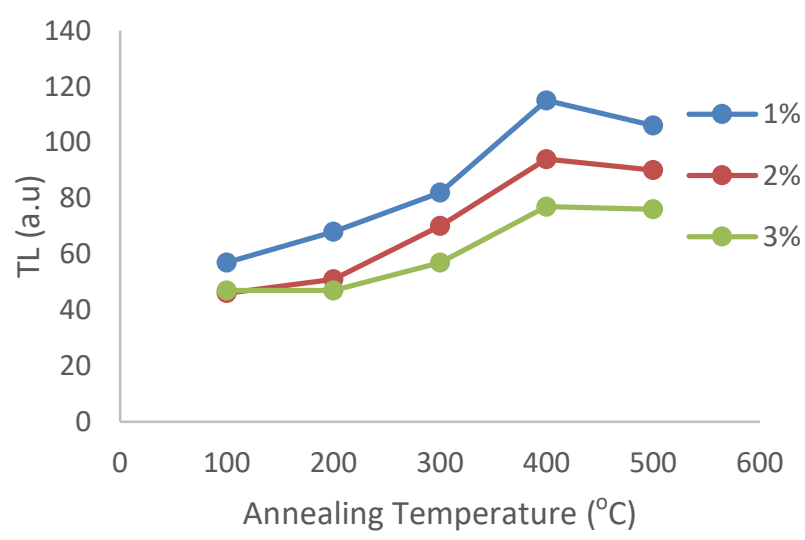

Fig.1. The behavior of $\mathrm{TL}$ response of $\mathrm{Mg}$-doped $\mathrm{ZnO}$ as function of annealing temperature for the three concentrations (annealing time $4 \mathrm{~h}$ ).

In order to check the number of cycles the $\mathrm{Mg}$-doped $\mathrm{ZnO}$ can be used without loss in sensitivity, seven samples were selected for each concentration five of them was irradiated to known dose and the other two kept in the lab.
The $\mathrm{Mg}$-doped $\mathrm{ZnO}$ samples was annealed at the optimum condition determined and irradiated with the same dose to measure the TL intensity. This process was repeated for 10 cycles for each concentration. No change in TL intensity or glow peak shape was observed even after the specified number of annealing cycles and the variation of TL intensity after different cycles of irradiation, annealing and reading out is illustrated in Fig. 2.

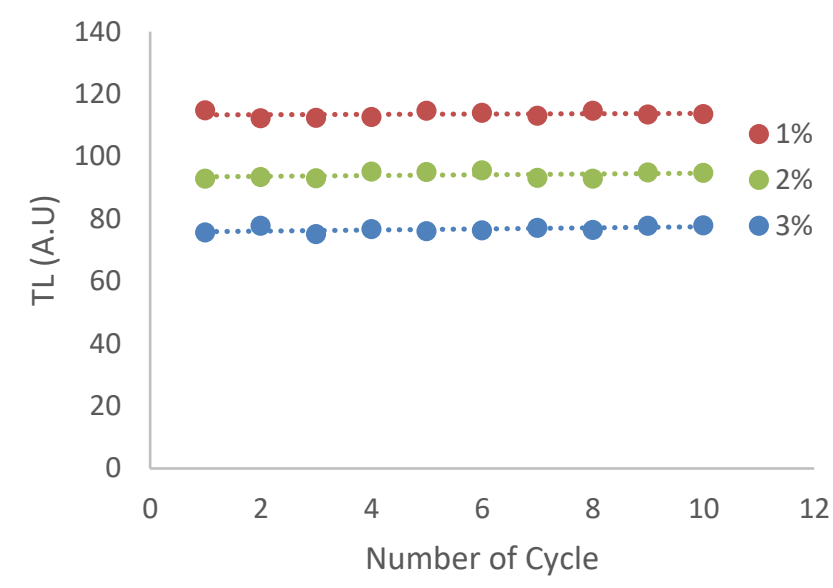

Fig. 2. Variation of TL sensitivity of $\mathrm{Mg}$-doped $\mathrm{ZnO}$ after different cycles of annealing and read out.

\section{B. Dose Response}

$\mathrm{TL}$ response was measured, as a function of X-ray radiation exposure of the three prepared concentrations of Mg-doped $\mathrm{ZnO}$. Fig 3 show plots of TL versus tube voltage when $\mathrm{mAs}$ are installed at $40 \mathrm{~mA}$. It was found that the response of $\mathrm{Mg}$-doped $\mathrm{ZnO}$ is linear up to the voltage used for the three concentrations. Fig 4 shows a plot of TL versus $\mathrm{mAs}$ when voltage is stabilized at $80 \mathrm{kV}_{\mathrm{P}}$, the figure shows that the TL response increases with increasing mAs of the three concentrations. The increase in the intensity of the glow curves with the increasing mAs can be understood by the fact that more and more traps responsible for these glow peaks are filled with the increased radiation. These traps release their own charge carriers upon thermal stimulation with their counterparts, resulting in different glow peaks.

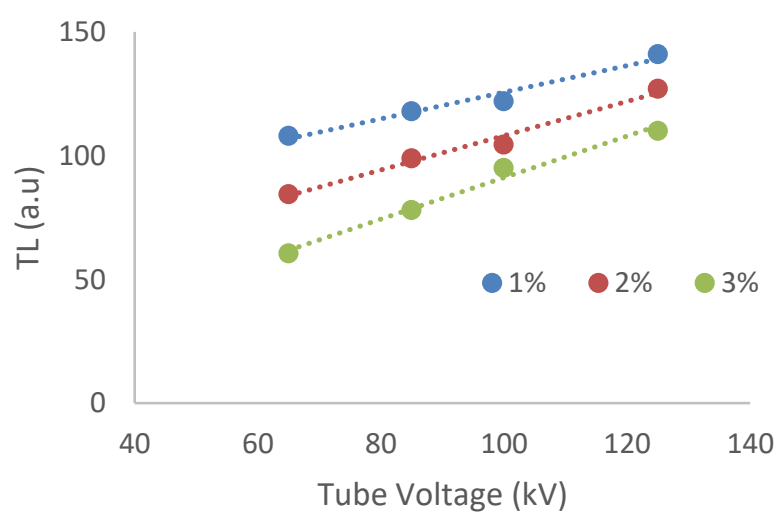

Fig.3. TL response versus tube voltage for $\mathrm{Mg}$-doped $\mathrm{ZnO}$ at fixed $\mathrm{mAs}$. 


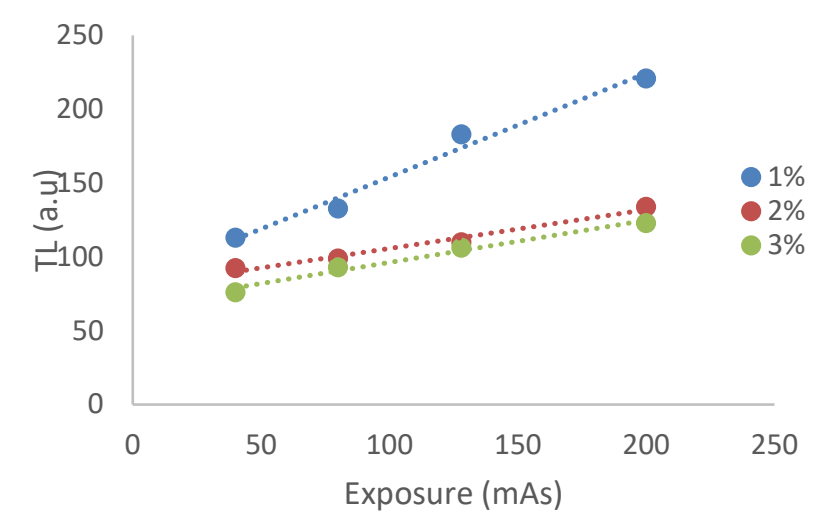

Fig.4. TL response versus exposure (mAs) for $\mathrm{Mg}$-doped $\mathrm{ZnO}$ at fixed $\mathrm{kV}_{\mathrm{p}}$.

\section{TL Fading}

The stability of the dosimeter storage after irradiation is important characteristics in selecting a material for dosimetry applications. Hence, it is necessary to assess whether the charges trapped in phosphorous before reading can be lost by thermal, optical or any other anomalous fading. The stability of the stored TL signal was studied over a 30-days using 40 samples in 8 groups per concentration. These groups were stored under the same conditions for different period of time after exposed to " $82 \mathrm{kV}_{\mathrm{p}}, 40 \mathrm{mAs}, 40 \mathrm{~cm} \mathrm{SSD"} \mathrm{X-ray.} \mathrm{It} \mathrm{was} \mathrm{found} \mathrm{that} \mathrm{the} \mathrm{TL}$ signal faded about $2.3 \%$ per day for $1 \%$ sample and about $2.7 \%$ per day for $2 \%$ sample, and about $2.2 \%$ per day for $3 \%$ sample.

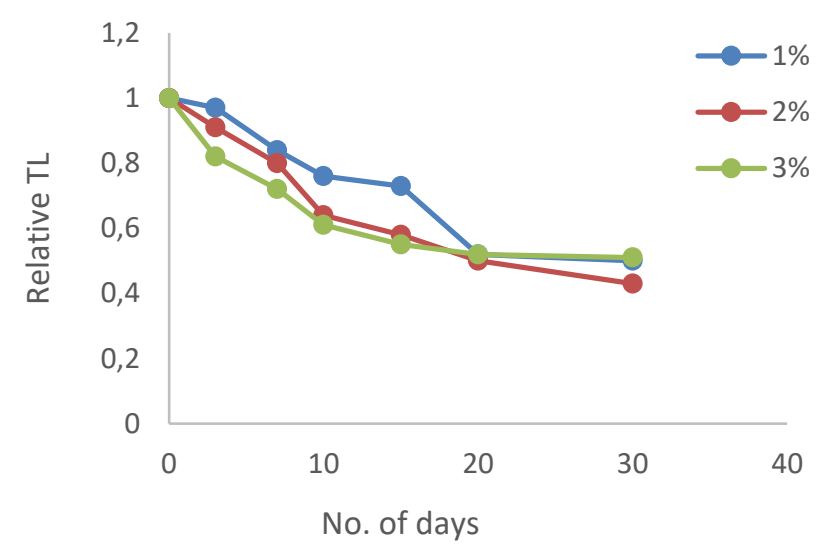

Fig.5. Fading characteristics of Mg-doped $\mathrm{ZnO}$ stored in the dark at ambient temperature.

\section{Effective Atomic Number}

The response of a TL phosphor to ionizing radiations (especially X-ray and Gamma ray) depends on the atomic number of the components and not on the chemical composition of these components. Hence, it is necessary to determine the effective atomic number $\left(Z_{\text {eff }}\right)$ of the $\mathrm{Mg}$ doped $\mathrm{ZnO}$ to know the response of the substance to radiations of different energy. Mayneord determined the effective atomic number $\mathrm{Z}_{\mathrm{eff}}$ of a compound by equation (1) [25].

$$
Z_{e f f}=\sqrt[2.94]{a_{1} z^{2.94}+a_{2} z^{2.94}+\ldots . .+a_{n} z^{2.94}}
$$

where $a_{1}, \ldots \ldots a_{n}$, are the fractional contributions of each element to the total number of electrons in the mixture. The effective atomic number of $\mathrm{Mg}$-doped $\mathrm{ZnO}$ was found to be 25.5, which have a higher effective atomic number compared to tissue. Higher atomic number materials generally result in high sensitivity to gamma radiation but needs suitable filters to compensate for over response at lower energy radiation, when used for personnel monitoring.

\section{E. TL Glow Curve Deconvolution (GCD) for Dose Parameter Evaluation}

The shape of the glow curve experimentally recorded shows, in fact, the superposition of many individual TL peaks, each associated with different types of local states.

The TL glow curves of $1 \%, 2 \%$ and $3 \% \mathrm{Mg}$-doped $\mathrm{ZnO}$ were deconvoluted using the kinematic expression of general order arrangement, namely:

$I=s n_{0} \exp \left(-\frac{E}{k T}\right)\left[1+\left(\frac{s(b-1)}{\beta}\right) \int_{T_{0}}^{T} \exp \left(-\frac{E}{k T}\right) d T^{\prime}\right]^{\frac{-b}{b-1}}$

where $\beta$ is the constant heating rate, $\mathrm{b}$ is the order of kinetic properties and $s=s^{\prime \prime} n_{0}{ }^{(b-1)}$. With this definition, s contains units of frequency $\left(\mathrm{s}^{-1}\right)$ similar to that in the first order kinetic, but it dependent on the applied dose rather than being fixed. Eq. (2) includes the second order kinetics $(b=2)$ and reduces to the first order kinematic parameters $(b=1)$ when $b$ is one of the unknown parameters, we decided to use general order kinetics with $b$ that may take any value between one and two. Fitting TL glow curves was performed using PeakFit [26], [27] software.

Fig. 6, 7, and 8 represent the deconvoluted graph for $\mathrm{ZnO}$ doped with $1 \%, 2 \%$ and $3 \% \mathrm{Mg}$ phosphors annealed at $400{ }^{\circ} \mathrm{C}$ irradiated with X-ray " $82 \mathrm{kVp}, 40 \mathrm{mAs}$ ".

The figures also show the characteristic glow curve with different $\mathrm{ZnO}: \mathrm{Mg}$ ratios. The curves showed a broad shape $\mathrm{TL}$ glow curve with a maximum located above $270{ }^{\circ} \mathrm{C}$. It is observed that the sensitivity of the curve increases at the ratio $\mathrm{ZnO}: \mathrm{Mg}(2 \%)$, yet TL signals being decrease by less than $\mathrm{ZnO}: \mathrm{Mg}$ (1 and $3 \%)$. This behavior is indicating a significant decrease in sensitivity with an increase in the $\mathrm{ZnO}: \mathrm{Mg}$ ratios. It may indicate a $\mathrm{TL}$ mechanism that includes at least two overlapping trap levels, one of which acts as a competitor and the other as the active trap.

Obviously, the addition of magnesium makes the TL glow curve wider and converts the entire TL complex to lower temperatures. The presence of magnesium did not introduce new traps as the three samples were fitted with different number of peaks. Its presence is mainly sensed by the values of the frequency factor as a one order increase in size is observed. The contrast of the TL intensity signal with focus is more sensitive in the case of $2 \% \mathrm{Mg}$-doped $\mathrm{ZnO}$. The effect of adding magnesium as a dopant on trap depth is fairly minimal without a change in the order of kinetics. Table 1 illustrates the trapping parameters for individual peaks for one sample of each types. It is interesting to note that all the individual peaks were fitted with general order kinetics, variation in the activation energy, and the frequency factor. This indicates that (1) the retrapping process dominates the recombination process in this material (2) the material may have very overlapping trapping levels with a distribution of frequency factors. 


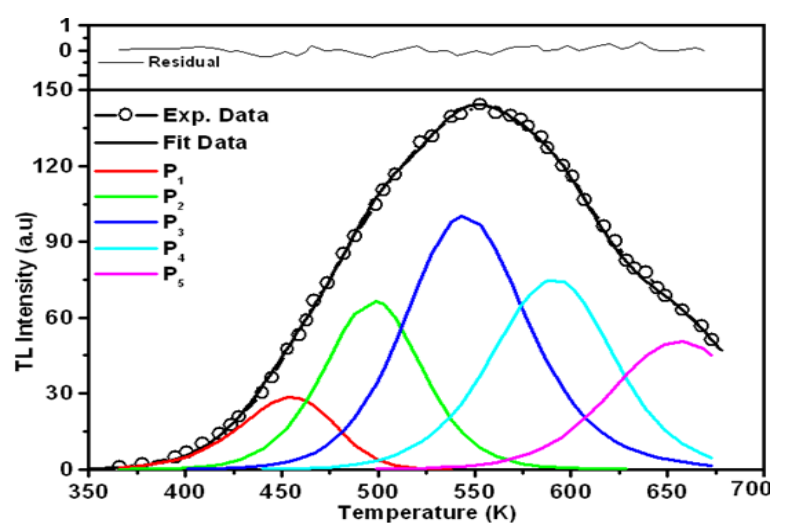

Fig. 6. TL glow curve deconvolution of $1 \% \mathrm{Mg}$-doped $\mathrm{ZnO}$ phosphor recorded after irradiation with X-ray " $82 \mathrm{kVp}, 40 \mathrm{mAs} "$.

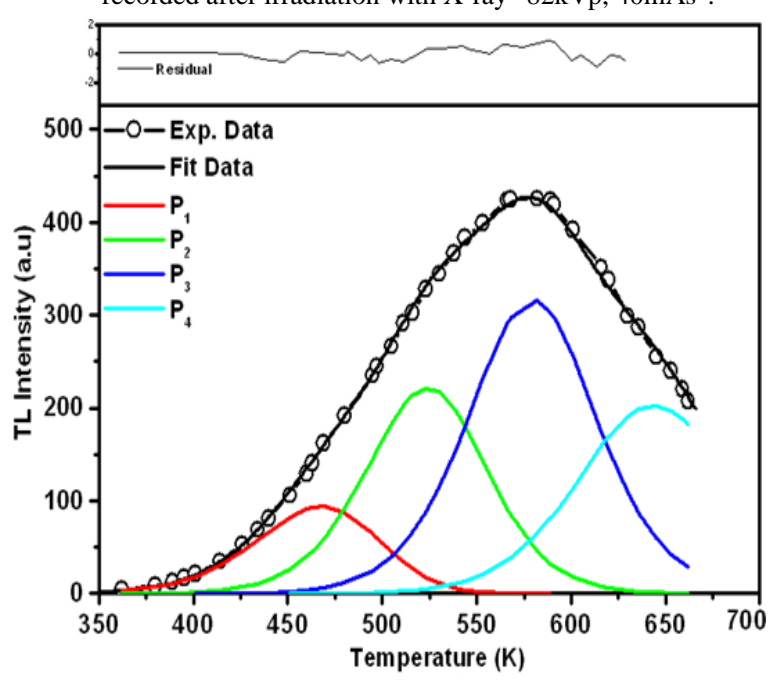

Fig. 7. TL glow curve deconvolution of $2 \% \mathrm{Mg}$-doped $\mathrm{ZnO}$ phosphor

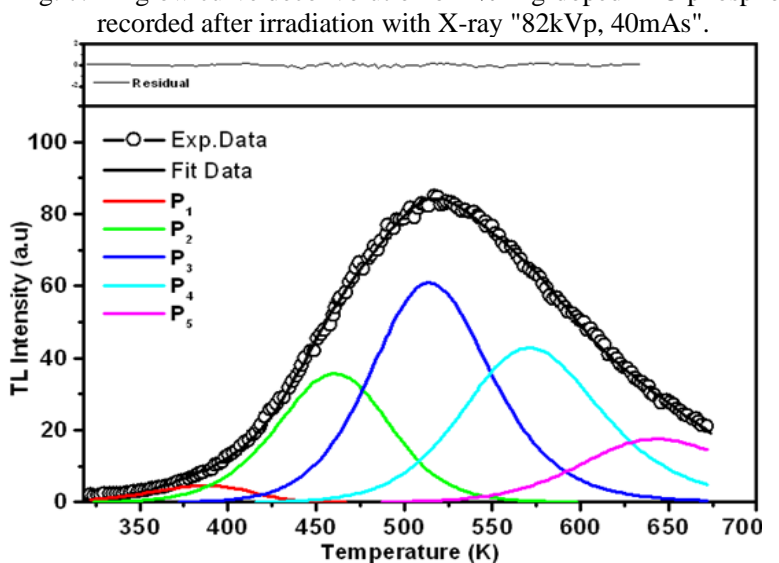

Fig. 8. TL glow curve deconvolution of $3 \% \mathrm{Mg}$-doped $\mathrm{ZnO}$ phosphor recorded after irradiation with X-ray "82kVp, 40mAs".

TABLE I: TL TRAPPING PARAMETERS FOR 1\%, $2 \%$ AND 3\% MG-DOPED ZNO SAMPLES OBTAINED BY CURVE ING

\begin{tabular}{ccccccc}
\hline \hline $\begin{array}{c}\text { Type of } \\
\text { Sample }\end{array}$ & $\begin{array}{c}\text { \# of } \\
\text { Traps }\end{array}$ & $\mathrm{E}(\mathrm{eV})$ & $\begin{array}{c}\mathrm{T}_{\mathrm{M}} \\
(\mathrm{K})\end{array}$ & $\mathrm{b}$ & $\mathrm{n}_{0}$ & $\mathrm{~s}\left(\mathrm{~s}^{-1}\right)$ \\
\hline \hline & $\mathrm{P}_{1}$ & 0.774 & 455 & 1.22 & $7.25 \times 10^{3}$ & $1.39 \times 10^{6}$ \\
$1 \% \mathrm{Mg}-$ & $\mathrm{P}_{2}$ & 1.059 & 498 & 1.64 & $1.78 \times 10^{4}$ & $2.45 \times 10^{8}$ \\
doped & $\mathrm{P}_{3}$ & 1.102 & 545 & 1.89 & $3.35 \times 10^{4}$ & $6.19 \times 10^{7}$ \\
$\mathrm{ZnO}$ & $\mathrm{P}_{4}$ & 1.168 & 591 & 1.57 & $2.49 \times 10^{4}$ & $3.28 \times 10^{6}$ \\
& $\mathrm{P}_{5}$ & 1.377 & 656 & 1.97 & $2.01 \times 10^{4}$ & $1.27 \times 10^{8}$ \\
\hline \multirow{2}{*}{$2 \% \mathrm{Mg}-$} & $\mathrm{P}_{1}$ & 0.609 & 468 & 1.22 & $3.15 \times 10^{4}$ & $1.10 \times 10^{4}$ \\
doped & $\mathrm{P}_{2}$ & 0.884 & 524 & 1.52 & $7.39 \times 10^{4}$ & $1.10 \times 10^{6}$ \\
$\mathrm{ZnO}$ & $\mathrm{P}_{3}$ & 1.07 & 579 & 1.65 & $1.126 \times 10^{5}$ & $6.95 \times 10^{6}$ \\
& $\mathrm{P}_{4}$ & 1.19 & 643 & 2.14 & $9.35 \times 10^{4}$ & $6.30 \times 10^{6}$ \\
\hline \multirow{2}{*}{$3 \% \mathrm{Mg}-$} & $\mathrm{P}_{1}$ & 0.446 & 388 & 1.09 & $1.35 \times 10^{3}$ & $2.11 \times 10^{3}$ \\
doped & $\mathrm{P}_{2}$ & 0.666 & 461 & 1.47 & $1.19 \times 10^{4}$ & $6.59 \times 10^{4}$ \\
$\mathrm{ZnO}$ & $\mathrm{P}_{3}$ & 0.885 & 514 & 1.83 & $2.19 \times 10^{4}$ & $1.67 \times 10^{6}$ \\
& $\mathrm{P}_{4}$ & 0.971 & 571 & 1.96 & $1.80 \times 10^{4}$ & $1.16 \times 10^{6}$ \\
\hline \hline
\end{tabular}

\section{CONCLUSION}

Pellet-shaped $\mathrm{Mg}$-doped $\mathrm{ZnO}$ samples were synthesized by Sol-Gel technique and exposed to X-ray irradiation to investigate their thermoluminescence features. The characteristic TL glow curves show a complex shape resolved in five individual TL peaks. There is no change in TL intensity or glow peak shape after different cycles of irradiation, anneal and read- out. The integrated TL as a function of the irradiation dose displays a linear dependence in the dose range used, the TL signal faded down from $2.2 \% /$ day to $2.7 \% /$ day for the three concentrations. The effective atomic number of $\mathrm{Mg}$-doped $\mathrm{ZnO}$ is 25.5 and the kinetics parameters of the individual peaks were estimated using the computerized glow curve deconvolution program. It is concluded that the synthesized and characterized $\mathrm{Mg}$ doped $\mathrm{ZnO}$ is a promising phosphor to develop TL dosimeters.

\section{REFERENCES}

[1] C. Furetta, Handbook of Thermoluminescence; World Scientific Pub: Singapore, (2003); ISBN: 10: 9812382402.

[2] M.Y. William; S. Shionoya; Yamamoto, H. Practical Applications of Phosphors; CRC Press: Boca Raton, FL, USA, (2007); ISBN 9781420043693. C. Y. Lin, M. Wu, J. A. Bloom, I. J. Cox, and M. Miller, "Rotation, scale, and translation resilient public watermarking for images", IEEE Trans. Image Process., vol. 10, no. 5, pp. 767-782, May 2001.

[3] M. Oberhofer, Applied Thermoluminescence Dosimetry; Adam Hilger: Bristol, TN, USA, (1979); ISBN 0852745443.

[4] R. Chen; S.W.S. McKeever, Theory of Thermoluminescence and Related Phenomena; World Scientific: Singapore, (1997); ISBN 0810222955.

[5] S.W.S. McKeever, Thermoluminescence of Solids; Cambridge University Press: Cambridge, UK, (1988); ISBN 0521368111.

[6] V. Pagonis; G. Kitis; C. Furetta, Numerical and Practical Exercises in Thermoluminescence; Springer: New York, NY, USA, (2006); ISBN 0387260633.

[7] R. Chen; V. Pagonis, Thermally and Optically Stimulated Luminescence Simulation Approach; JohnWiley: Chichester, UK, (2011); ISBN 9780470749272.

[8] V. Etacheri. R. Roshan, and V. Kumar, "Mg-Doped ZnO Nanoparticles for Efficient Sunlight-Driven Photocatalysis "ACS Appl. Mater. Interfaces 4, 2717 (2012).

[9] U. Ozgur, Y. I. Alivov, C. Liu. A. Teke. M. A. Reshchikov. S. Dogan V. Avrutin S. J. Cho, and H. Morkoc." A Comprehensive review of ZnO materials and Devices" J. Appl Phys. 98, 041301 (2005).

[10] E. R. Leite. A. P. Maciel. I. T. Weber. P. N. Lisboa-Filho. E. Longe. C. O. Paiva-Santos. A. V. C. Andrade. C. A Pakoscimas. Y. Maniette, and W. H. Schreiner. Development of Metal Oxide Nanoparticles with High Stability against Particle Growth using a Metastable Solid solution" Adv. Mater. 14. 905 (2002).

[11] H.C. Hsu.; C. Y. Wu; H.M. Cheng; W.F. Hsieh, "Band gap engineering and stimulated emission of $\mathrm{ZnMgO}$ nanowires ". Appl. Phys. Lett. 89, (2006).

[12] J. Peng, J. Guo; S. Ding; Q. Xu; H. Li; X. Tan; X. Zhao, " Preparation and Properties of Ternary ZnMgO Nanowires" Rare Met. 30, 292-297 (2011).

[13] H. Zhuang; J. Wang; H. Liu; J. Li; P. Xu, "Structural and optical Properties of $\mathrm{ZnO}$ Nanowires Doped with Magnesium" Acta Phys. Pol. A. 119(6), 819-823 (2011).

[14] J.Y. Cho; I.K. Kim; I.O. Jung; J.H. Mooon; J. H. Kim, " Effects of $\mathrm{Mg}$ doping concentration on the band gap of $\mathrm{ZnO} / \mathrm{MgxZn1}-\mathrm{xO}$ multilayer thin films prepared using pulsed laser deposition method" J. Electrocerom. 23, 442-446 (2009).

[15] Abeer. Z. Abraheem and Y.A. Abdulla. Preparation and Characterization of the Thermoluminescence Properties of Mg-doped ZnO. IJSBAR. 49(1), 133-142 (2020).

[16] A. Burlacu, V.V. Ursaki, V.A. Skuratov, et al. The impact of morphology upon the radiation hardness of $\mathrm{ZnO}$ layers. Nanotechnology 19:215714. (2008).

[17] C. Cruz-Va'zquez, S.E. Burruel-Ibarra, H. Grijalva-Monteverde, et al. Thermally and optically stimulated luminescence of new $\mathrm{ZnO}$ 
nanophosphors exposed to beta particle irradiation. Radiat. Eff. Defects Solids 162:737-743. (2007).

[18] C. E. Secu, M. Sima, Photoluminescence and thermoluminescence of $\mathrm{ZnO}$ nano-needle arrays and films. Opt Mater (Amst) 31:876-880. (2009).

[19] D. Sahu, B.S. Acharya, B.P. Bagm et al. Probing the surface states in nanoZnO powder synthesized by sonication method: photo and thermo-luminescence studies. J Lumin 130:1371-1378. (2010).

[20] P.P. Pal, J. Manam, Evaluation of kinetics parameters in the Xirradiated TSL studies of RE3?-doped $(\mathrm{RE}=\mathrm{Eu}, \mathrm{Tb}) \mathrm{ZnO}$ nanorods for dosimetric applications. Radiat. Phys. Chem 88:7-13. (2013).

[21] A.J. Reddy, M.K. Kokila, H. Nagabhushana, et al. Structural, EPR, photo and thermoluminescence properties of $\mathrm{ZnO}$ : Fe nanoparticles. Mater Chem. Phys 133:876-883. (2012).

[22] J.T. Randall; M. H. F. Wilkins, The Phosphorescence of Various Solids. Proc. R. Soc. A Math. Phys. Eng. Sci. (1945), 184, 347-364.

[23] G.F.J. Garlick; A.F. Gibson, The Electron Trap Mechanism of Luminescence in Sulphide and Silicate Phosphors. Proc. Phys. Soc. (1948), 60, 574-590.

[24] M. S. Rasheedy, On the general-order kinetics of the thermoluminescence glow peak. J. Phys. Condens. Matter. (1993), 5, 633-636.

[25] P. R. Gonzales, C. Furetta, B. E. Calvo, M. I. Gaso, E. Cruz Zaragoza, "Dosimetric characterization of a new preparation of BaSO4 activated by Eu ions " Nucl. Instrum. Methods in Phys. Res. Sect., B260 .685692. (2007).

[26] M.H.A. Mhareb, M. Maghrabi, Y.S.M. Alajerami, S. Hashim, S.K. Ghoshal, M.A. Saleh, K.M. Abushab. Glow curve analysis of glassy system dosimeter subjected to photon and electron irradiations. Results in Physics 10 (2018) 772-776.

[27] F. Khamis and D. -E. Arafah. Improved Thermoluminescence Properties of Natural $\mathrm{NaCl}$ Salt Extracted from Mediterranean Sea Water Relevant to Radiation Dosimetry. EJ-Appl. Phys. 2020. 